\title{
Psoriasis and Pregnancy: Retrospective Evaluation of 47 Pregnancies in a Tertiary Center
}

\author{
Efsun TANACAN 1 , Atakan TANACAN ${ }^{2}$, Erdem FADILOGLU ${ }^{2}$, Canan UNAL ${ }^{2}$, M. Sinan BEKSAC ${ }^{2}$ \\ Ankara, Turkey
}

\section{ABSTRACT}

OBJECTIVE: To evaluate the impact of psoriasis on pregnancy outcomes.

STUDY DESIGN: Data of pregnant women with chronic plaque psoriasis who were followed up at Hacettepe University Hospital between January 1, 2010 and December 31, 2017 were evaluated. Pregnant women with singleton pregnancies who had chronic plaque psoriasis were included in the study. Patients were divided into two groups based on the clinical course of psoriasis: group 1 (improvement/disease-stable), and group 2 (deterioration). Median maternal age, gravida, parity, gestational week at birth, birthweight, $5^{\text {th }}$ minute Apgar score together with the rates of cesarean section, neonatal intensive care unit admission and pregnancy complications (spontaneous abortion, preterm delivery, fetal growth restriction and preeclampsia) were compared between the groups.

RESULTS: There were 29 (61.7\%) patients in group 1 and 18 (38.3\%) patients in group 2. Mean values for maternal age, gravida and parity were comparable between the groups ( $p$ values were $0.32,0.09$ and 0.17 , respectively). Median values for gestational week at birth (39.2 vs $36.1, p=0.002)$, birthweight (3200 vs $2310, p=0.002$ ) and $5^{\text {th }}$ minute Apgar score (9 vs $7, p<0.001$ ) were statistically significantly lower in group 2. Cesarean section (33.3\% vs $71.4 \%, p=0.02)$, neonatal intensive care unit admission $(11.1 \%$ vs $64.3 \%, p<0.001)$ and pregnancy complication rates $(p=0.003)$ were statistically significantly higher in group 2. Frequencies of spontaneous abortion, preterm delivery, fetal growth restriction and preeclampsia were $6.9 \%, 10.3 \%, 3.4 \%$ and $3.4 \%$ in group 1 , and $22.2 \%, 27.8 \%, 16.7 \%$ and $16.7 \%$ in group 2, respectively.

CONCLUSION: Deterioration of psoriasis in pregnancy was associated with adverse obstetric outcome.

Keywords: Adverse obstetric outcome, Autoimmune disease, Placental inflammation, Pregnancy, Psoriasis

Gynecol Obstet Reprod Med 2019;25(3):128-132

\section{Introduction}

Psoriasis is a chronic autoimmune skin disease that affects approximately $2 \%$ of the population (1). It is commonly char-

\footnotetext{
${ }^{1}$ Department of Dermatology and Venereology Ankara Training and Research Hospital, Ankara

${ }^{2}$ Division of Perinatology Department of Obstetrics and Gynecology Hacettepe University Hospital, Ankara
}

Address of Correspondence: Atakan Tanacan

Division of Perinatology, Department of Obstetrics and Gynecology, Hacettepe

University Hospital, 06100 Ankara,

Turkey

atakantanacan@yahoo.com

Submitted for Publication: 09.10 .2018

Revised for: Publication

13.10.2018

26.10.2018

ET: 0000-0003-1975-7460,

Ss of the author

EF: 0000-0001-7953-2517,

AT: 0000-0001-8209-8248,

MSB: 0000-0001-6362-787X

\begin{tabular}{|c|c|}
\hline Quick Response Code: & Access this article online \\
\cline { 2 - 2 } & $\begin{array}{l}\text { Website: www.gorm.com.tr } \\
\text { e- mail: info@gorm.com.tr }\end{array}$ \\
\cline { 2 - 3 } & DOI: $10.21613 /$ GORM.2018.850 \\
\hline
\end{tabular}

How to cite this article: Tanacan E. Tanacan A.Fadiloglu E. Unal C. Beksac MS. Psoriasis and Pregnancy: Retrospective Evaluation of 47 Pregnancies in a Tertiary Center. Gynecol Obstet Reprod Med. 2019;25(3):128-132 acterized by well-demarcated, erythematous plaques with silver scales (2). The lesions occur mostly on the extensor surface of the elbows, knees or the scalp $(1,2)$. Approximately half of the patients are women and the majority of them have onset of disease before the age of 40 (2). Therefore, psoriasis may complicate pregnancies, mainly due to its epidemiologic characteristics.

The major clinical forms of psoriasis are: chronic plaque psoriasis, guttate psoriasis, pustular psoriasis, erythrodermic psoriasis, inverse psoriasis and nail psoriasis (3). Chronic plaque psoriasis is the most common variant and pustular psoriasis may lead to life-threatening complications (3).

Although, the exact pathophysiology of the disease has not yet been elucidated, T lymphocytes and dendritic cells are hypothesized to play a pivotal role in the pathogenesis (4). Hyperproliferation and abnormal differentiation of the epidermis, infiltration of inflammatory cells together with vascular dilatation are responsible for the clinical findings and symptoms of psoriasis (4). Genetic predisposition, smoking, obesity, medications, infections, alcohol consumption, vitamin D deficiency and stress are the most common risk factors for the onset of psoriasis (5). Patients with psoriasis are at increased risk for arthritis, cardiovascular disease, malignancy, diabetes, 
hypertension, metabolic syndrome, inflammatory bowel disease, serious infections and autoimmune disorders (6-8).

Emollients, topical corticosteroids, vitamin D analogs, calcipotriene, calcitriol, tazarotene, calcineurin inhibitors, anthralin, narrowband ultraviolet B (UVB), methotrexate, apremilast and biological agents are the treatment options for psoriasis depending on the severity of the disease (9). However, no curative treatment has yet been discovered (9).

The course of psoriasis during pregnancy is usually unpredictable. Psoriasis improves during pregnancy in 40 to 60 percent of women, worsens in 10 to 20 percent, and remains stable in the remainder $(1,10)$. Relatively higher rates of improvement may be associated with oestrogen/progesteronemediated immunosuppression during pregnancy $(1,10)$. Although increased rates of spontaneous abortion, preterm birth, low birthweight, fetal growth restriction (FGR) and cesarean section (CS) were reported in some studies, the knowledge on the relationship between psoriasis and pregnancy outcomes is limited $(1,2)$. Furthermore, choosing the most appropriate treatment protocols during pregnancy is another important issue for physicians (11). Topical therapy and narrowband UVB are the preferred treatment modalities in this period (11).

The aim of this study was to evaluate the impact of psoriasis on pregnancy outcomes at a tertiary center.

\section{Material and Method}

The data of pregnant women with chronic plaque psoriasis who were followed up at the Division of Perinatology, Hacettepe University Hospital, Ankara between January 1, 2010 and December 31, 2017 were evaluated in this retrospective cohort study. Necessary data were drawn from the "perinatology database".

Pregnant women with singleton pregnancies who had chronic plaque psoriasis were included in the study. Pregnant women with other clinical forms of psoriasis were excluded from the study.

The diagnosis of psoriasis was made according to physical examination, patients' history and skin biopsy in necessary cases by dermatologists (12). Severity and activation of disease during pregnancy was assessed with physical examination and the Psoriasis Area and Severity Index (PASI, ranging from 0 to 72), which takes into account appearance and extent of the lesions (13). Topical corticosteroids, emollients, moisturizers and narrowband UVB phototherapy in severe cases were applied to patients with deterioration of the disease.

Spontaneous abortion was defined as a clinically recognized pregnancy loss before the 20th week of gestation (14). Preterm delivery was defined as delivery that occured before 37 weeks of gestation (15). Fetal growth restriction (FGR) was defined as $<10^{\text {th }}$ percentile weight for gestational age on a singleton growth curve depending on sonographic biometric measurements (16). Preeclampsia was defined as the new onset of hypertension and significant end-organ dysfunction with or without proteinuria after 20 weeks of gestation in a previously normotensive woman (17).

Pregnant women were divided into two groups based on the clinical course of psoriasis during pregnancy: group 1 (improvement/disease-stable), and group 2 (deterioration). Median maternal age, gravida, parity, gestational week at birth, birthweight, $5^{\text {th }}$ minute Apgar score together with the rates of $\mathrm{CS}$, neonatal intensive care unit (NICU) admission and pregnancy complications (spontaneous abortion, preterm delivery, FGR and preeclampsia) were compared between the groups.

Statistical analyses were performed using the Statistical Package for the Social Sciences (SPSS.22, IBM SPSS Statistics for Windows, Version 22.0 Armonk, NY: IBM Corp.). Variables were investigated using visual (histograms, probability plots) and analytical methods (Shapiro-Wilk's test) to determine whether they were normally distributed. Descriptive analyses are presented as medians and interquartile range for non-normally distributed variables. As continuous variables were not normally distributed, the MannWhitney $\mathrm{U}$ test was conducted to compare the median values among groups. Categorical variables were defined based on numbers and percentages. Chi-square test was used to compare categorical variables among the groups. A two-tailed $\mathrm{P}$ value $<0.05$ was regarded as statistically significant.

The study protocol was approved by the Hacettepe University Ethics Committee (GO 18/326).

\section{Results}

Improvement of psoriasis was observed in 21 (44.7\%) patients. The course of the disease was stable in $8(17 \%)$ patients and deterioration was reported in $18(38.3 \%)$ patients. There were 29 patients in group 1 and 18 patients in group 2 . Topical corticosteroids, emollients, and moisturizers were applied to all patients with activation of the disease. Additionally, narrowband UVB phototherapy were applied to 7 patients with severe lesions in group 2.

Mean values for maternal age, gravida, and parity were comparable between the groups ( $\mathrm{p}$ values were $0.32,0.09$ and 0.17 , respectively). On the other hand, median values for gestational week at birth (39.2 vs $36.1, p=0.002)$, birthweight ( 3200 vs $2310, p=0.002)$ and $5^{\text {th }}$ minute Apgar score (9 vs 7 , $p<0.001)$ were statistically significantly lower in group 2 compared to group 1. Additionally, CS (33.3\% vs $71.4 \%$, $p=0.02$ ), NICU admission ( $11.1 \%$ vs $64.3 \%, p<0.001)$ and pregnancy complication rates $(p=0.003)$ were statistically significantly higher in group 2 . The frequencies of spontaneous abortion, preterm delivery, FGR, and preeclampsia were $6.9 \%, 10.3 \%, 3.4 \%$ and $3.4 \%$, respectively in group 1 . However, frequencies of the same parameters in group 2 were $22.2 \%, 27.8 \%, 16.7 \%$ and $16.7 \%$, respectively (Table I). 
Table I: Comparision of the patients for demographic features, clinical characteristics and obstetric outcomes according to the course of psoriasis during pregnancy

\begin{tabular}{|c|c|c|c|}
\hline & $\begin{array}{c}\text { Improvement/ } \\
\text { disease-stable group } \\
(n=29)\end{array}$ & $\begin{array}{l}\text { Deterioration } \\
\text { group } \\
(n=18)\end{array}$ & $\begin{array}{c}p \\
\text { value }\end{array}$ \\
\hline Maternal age (years) $)^{a, b}$ & $29(3)$ & $28(4)$ & 0.32 \\
\hline Gravida & $2(1)$ & $2(1)$ & 0.09 \\
\hline Paritya,b & $1(1)$ & $1(1)$ & 0.17 \\
\hline Gestational week at birtha,b & $39.2(1.9)$ & $36.1(2.0)$ & 0.002 \\
\hline Birthweight $(\mathrm{g})^{\mathrm{a}, \mathrm{b}}$ & $3200(330)$ & $2310(825)$ & 0.002 \\
\hline Apgar score $\left(5^{\text {th }} \text { minute }\right)^{a, b}$ & $9(1)$ & $7(1)$ & $<0.001$ \\
\hline Cesarean section rate ${ }^{c}$ & $33.3 \%$ & $71.4 \%$ & 0.02 \\
\hline NICU admissionc & $11.1 \%$ & $64.3 \%$ & $<0.001$ \\
\hline \multicolumn{4}{|l|}{ Pregnancy complicationc } \\
\hline Spontaneous abortion & $6.9 \%$ & $22.2 \%$ & \multirow{4}{*}{0.003} \\
\hline Preterm delivery & $10.3 \%$ & $27.8 \%$ & \\
\hline$F G R$ & $3.4 \%$ & $16.7 \%$ & \\
\hline Preeclampsia & $3.4 \%$ & $16.7 \%$ & \\
\hline
\end{tabular}

FGR: Fetal growth restriction, NICU: Neonatal intensive care unit, a: median, interquartile range values, $b$ : Mann-Whitney $U$ test was performed, c: Chi-square test was performed

\section{Discussion}

This study demonstrated that deterioration of psoriasis in pregnancy was associated with adverse obstetric outcomes. Gestational week at birth, birthweight and $5^{\text {th }}$ minute Apgar score were found to be lower in the deterioration group compared to the improvement/disease stable group. Additionally, increased rates of CS, NICU admission and pregnancy complications were observed in the deterioration group.

The relationship between autoimmune diseases and adverse pregnancy outcomes were reported in various studies (18-21). Autoimmune antibodies, cell degradation products and inflammatory cytokines were most likely responsible for the injury to vascular structures of the placenta and cellular components of the maternal fetal interface, resulting in "placental inflammation". Altered biological events and immune system activation might result in impaired implantation and disturbed fetal perfusion (18-21). Similar to other autoimmune diseases, psoriasis is characterized by systemic immune dysregulation related to proinflammatory cytokines $(1,22)$. Increased activity of Thelper 1 and T-helper 17 cells in the immunopathogenesis of psoriasis were discussed in the current literature $(1,3)$. T-helper 1 related proinflammatory cytokines interleukin 2 and interferon- $\gamma$ take part in implantation $(1,23)$. Furthermore, interleukin $17 \mathrm{~A}$, which is produced by T-helper 17 cells, plays a protective role from invading pathogens in the decidua $(1,23)$. Thus, psoriasis and pregnancy may affect each other by common immunological pathways.

There are a limited number of studies in the literature evaluating the relationship between psoriasis and pregnancy $(1,2$, 24). Usually, an improvement in the course of the disease was reported in pregnant women, most probably due to hormonal changes in pregnancy resulting in an immune tolerant state $(10,25)$. Improvement of psoriasis was observed in $21(44.7 \%)$ patients in this study, which was consistent with the literature $(10,25)$. Another important point is the choice of treatment during pregnancy, since some medications are considered contraindicated (26). Topical therapies and narrowband UVB phototherapy in severe cases were applied to patients with deterioration of the disease at our institution. No biological agents were used due to limited data on the safety of these medications during pregnancy. However, there are a growing number of studies in the literature demonstrating that biological agents may be used safely during pregnancy $(27,28)$.

The relationship between psoriasis and pregnancy outcomes is unclear (1). According to a recent systematic review of observational studies, increased risk for at least one adverse fetal outcome (spontaneous abortion, CS, low birth weight, macrosomia, large-for-gestational age, or a composite outcome that included prematurity and low birth weight) was reported in four of the nine included studies (1). However, the study results were inconsistent (1). Systemic immuno-mediated inflammatory processes in psoriasis lead to increased levels of C-reactive protein, fibrinogen and other inflammatory cytokines (2). Inflammation was reported to be associated with adverse pregnancy outcomes such as preterm birth, low birth weight and preeclampsia $(29,30)$. Therefore, a higher rate of pregnancy complications in the disease deterioration group was most probably due to the increased systemic inflammatory response in this group. Additionally, increased rates of CS and NICU admission were related to the higher frequencies of preterm delivery, FGR and preeclampsia in this study. 
The main strengths of this study were the single-center experience and a multidisciplinary approach. On the other hand, the limitations of the study were the relatively small number of patients and the retrospective design.

In conclusion, deterioration of psoriasis in pregnancy was associated with adverse obstetric outcomes. Thus, physicians should be cautious in the management of pregnant women with psoriasis, especially in cases with activation of the disease.

Acknowledgments: Special thanks to all of the medical staff in the perinatology and dermatology clinics for their contribution in providing optimal healthcare for the patients.

Funding: No funding was used for this study.

Conflict of interest statement: The authors state no conflict of interest.

Author Contributions: ET: Concept, data processing, analysis, and interpretation, manuscript writing. AT: Data collection, processing, analysis, and interpretation, manuscript writing. EF: Data collection and processing, statistical analysis, manuscript writing. CU: Data collection, interpretation, manuscript writing. MSB: Supervision, concept, design, analysis and interpretation.

\section{References}

1. Bobotsis R, Gulliver WP, Monaghan K, Lynde C, Fleming P. Psoriasis and adverse pregnancy outcomes: A systematic review of observational studies. Br J Dermatol. 2016;175(3):464-72.

2. Lima XT, Janakiraman V, Hughes MD, Kimball AB. The impact of psoriasis on pregnancy outcomes. J Invest Dermatol. 2012;132(1):85-91.

3. Harden JL, Krueger JG, Bowcock AM. The immunogenetics of psoriasis: a comprehensive review. J Autoimmun. 2015;64:66-73.

4. Krueger J, Bowcock A. Psoriasis pathophysiology: current concepts of pathogenesis. Ann Rheum Dis. 2005;64 (suppl 2):ii30-ii6.

5. Huerta C, Rivero E, Rodríguez LAG. Incidence and risk factors for psoriasis in the general population. Arch Dermatol. 2007;143(12):1559-65.

6. Pearce DJ, Morrison AE, Higgins KB, Crane MM, Balkrishnan R, Fleischer AB, Jr., et al. The comorbid state of psoriasis patients in a university dermatology practice. J Dermatolog Treat. 2005;16(5-6):319-23.

7. Brauchli YB, Jick SS, Miret M, Meier CR. Psoriasis and risk of incident cancer: an inception cohort study with a nested case-control analysis. J Invest Dermatol. 2009;129(11):2604-12.

8. Wu JJ, Nguyen TU, Poon KY, Herrinton LJ. The association of psoriasis with autoimmune diseases. J Am Acad Dermatol. 2012;67(5):924-30.
9. Hamilton MP, Ntais D, Griffiths CE, Davies LM; Identification and Management of Psoriasis-Associated ComorbidiTy (IMPACT) Team. Psoriasis treatment and management-a systematic review of full economic evaluations. Br J Dermatol. 2015;172(3):574-83.

10. Murase JE, Chan KK, Garite TJ, Cooper DM, Weinstein GD. Hormonal effect on psoriasis in pregnancy and post partum. Arch Dermatol. 2005;141(5):601-6.

11. Danesh MJ, Murase JE. The new US Food and Drug Administration pregnancy and lactation labeling rules: Their impact on clinical practice. J Am Acad Dermatol. 2015;73(2):310-1.

12. Bernhard JD. Auspitz sign is not sensitive or specific for psoriasis. J Am Acad Dermatol. 1990;22(6 Pt 1):1079-81.

13. Mattei PL, Corey KC, Kimball AB. Psoriasis Area Severity Index (PASI) and the Dermatology Life Quality Index (DLQI): the correlation between disease severity and psychological burden in patients treated with biological therapies. J Eur Acad Dermatol Venereol. 2014; 28(3): $333-7$.

14. Regan L, Rai R. Epidemiology and the medical causes of miscarriage. Baillieres Best Pract Res Clin Obstet Gynaecol. 2000;14(5):839-54.

15. Sabour S. Prediction of preterm delivery using levels of VEGF and leptin in amniotic fluid from the second trimester: prediction rules. Arch Gynecol Obstet. 2015;291(4):719.

16. Benton SJ, McCowan LM, Heazell AE, Grynspan D, Hutcheon JA, Senger C, et al. Placental growth factor as a marker of fetal growth restriction caused by placental dysfunction. Placenta. 2016;42:1-8.

17. Zeisler H, Llurba E, Chantraine F, Vatish M, Staff AC, Sennström M, et al. Predictive value of the sFlt-1: PlGF ratio in women with suspected preeclampsia. N Engl J Med. 2016;374(1):13-22.

18. Beksaç K, Örgül G, Çağan M, Karaağaoğlu E, Arslan S, Beksaç MS. Retrospective evaluation of pregnant women with celiac disease. J Turk Ger Gynecol Assoc. 2017; 18(1):56-9.

19. Beksac K, Orgul G, Can GS, Oktem A, Kav T, Beksac MS. Management of inflammatory bowel disease and pregnancy using prophylactic low dose low molecular weight heparin and corticosteroids. J Clin Diagn Res. 2017;11(11)::QR01-QR03.

20. Orgul G, Aktoz F, Beksac MS. Behcet's disease and pregnancy: what to expect? J Obstet Gynaecol. 2018;38(2): 185-8.

21. Orgul G, Ozkan EU, Celik HT, Beksac MS. Autoimmune hepatitis and pregnancy: report of two cases with different maternal outcomes. Clin Exp Hepatol. 2017;3(4):212-4.

22. De Simone C, Caldarola G, Corbeddu M, Moro F, Tropea A, Moretta G, et al. A possible role of polycystic ovary syndrome for pregnancy complications in women with 
psoriasis. Drug Dev Res. 2014;75 Suppl 1:S64-6.

23. Saito S, Nakashima A, Shima T, Ito M. Th1/Th2/Th17 and regulatory $\mathrm{T}$ cell paradigm in pregnancy. Am $\mathrm{J}$ Reprod Immunol. 2010;63(6):601-10.

24. Bandoli G, Johnson DL, Jones KL, Lopez Jiminez J, Salas E, Mirrasoul N, et al. Potentially modifiable risk factors for adverse pregnancy outcomes in women with psoriasis. Am J Reprod Immunol. 2010;163(2):334-9.

25. Boyd AS, Morris LF, Phillips CM, Menter MA. Psoriasis and pregnancy: hormone and immune system interaction. Int J Dermatol. 1996;35(3):169-72.

26. Briggs GG. Drugs In Pregnancy And Lactation, For Pda: A Reference Guide To Fetal And Neonatal Risk: Lippincott Williams \& Wilkins; 2005.

27. Mahadevan U, Wolf DC, Dubinsky M, Cortot A, Lee SD, Siegel CA, et al. Placental transfer of anti-tumor necrosis factor agents in pregnant patients with inflammatory bowel disease. Clin Gastroenterol Hepatol. 2013;11(3): 286-92.

28. Cheent K, Nolan J, Shariq S, Kiho L, Pal A, Arnold J. Case report: fatal case of disseminated BCG infection in an infant born to a mother taking infliximab for Crohn's disease. J Crohns Colitis. 2010;4(5):603-5.

29. Sorokin Y, Romero R, Mele L, Wapner RJ, Iams JD, Dudley DJ, et al. Maternal serum interleukin-6, C-reactive protein, and matrix metalloproteinase- 9 concentrations as risk factors for preterm birth $<32$ weeks and adverse neonatal outcomes. Am J Perinatol. 2010;27(8):631-40.

30. Amarilyo G, Oren A, Mimouni F, Ochshorn Y, Deutsch V, Mandel D. Increased cord serum inflammatory markers in small-for-gestational-age neonates. J Perinatol. 2011;31 (1):30-2.

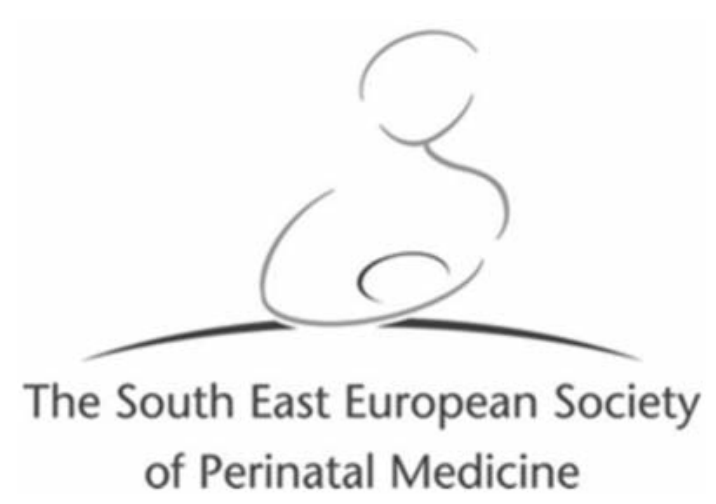

$8^{\text {th }}$ Congress of South-East European Society of Perinatal Medicine March 6-7, 2020 - Hotel Crowne Plaza

Athens, Greece

Correspondance:

Prof. Nikolaos Papantoniou (President)

Niki Pandi (Event coordinator)

118 Alex. Panagouli Str., Agia Paraskevi, Greece, PC 15343

+302106074200

+302106074203 (Direct)

+30 6973432013 (Mobile)

np@mdcongress.gr

www.mdcongress.gr 